\title{
Tracking the recovery of consciousness from coma
}

\author{
Steven Laureys, Mélanie Boly, and Pierre Maquet \\ Cyclotron Research Center and Department of Neurology, University of Liège, Liège, Belgium.
}

\begin{abstract}
Predicting the chances of recovery of consciousness and communication in patients who survive their coma but transit in a vegetative state or minimally conscious state (MCS) remains a major challenge for their medical caregivers. Very few studies have examined the slow neuronal changes underlying functional recovery of consciousness from severe chronic brain damage. A case study in this issue of the JCI reports an extraordinary recovery of functional verbal communication and motor function in a patient who remained in MCS for 19 years (see the related article beginning on page 2005). Diffusion tensor MRI showed increased fractional anisotropy (assumed to reflect myelinated fiber density) in posteromedial cortices, encompassing cuneus and precuneus. These same areas showed increased glucose metabolism as studied by PET scanning, likely reflecting the neuronal regrowth paralleling the patient's clinical recovery. This case shows that old dogmas need to be oppugned, as recovery with meaningful reduction in disability continued in this case for nearly 2 decades after extremely severe traumatic brain injury.
\end{abstract}

Forty years ago, Plum and Posner published the first edition of their landmark book, The diagnosis of stupor and coma (1). Since then, clinical research efforts in the field of acute brain damage and coma have increased nearly exponentially. Comatose patients who survive thanks to ever-improving intensive care medicine classically begin to awaken and recover consciousness within some days to weeks or will enter a vegetative state (VS). The clinical criteria of this diagnostic entity of "wakefulness without awareness" were first set out by Jennet and Plum in their 1972 milestone paper (2). The VS may be a transitional state on the route to further recovery or, unlike coma, which virtually never becomes chronic, the VS may progress to a long-standing, sometimes irreversible condition. Permanent VS, a prognostic term to be used with great caution, implies the prediction that the patient will not recover. It was introduced by the Multi-Society Task Force on PVS (persistent vegetative state) to denote irreversibility of the condition after 3 months following a nontraumatic brain injury

Nonstandard abbreviations used: MCS, minimally conscious state; $\mathrm{VS}$, vegetative state.

Conflict of interest: The authors have declared that no conflict of interest exists.

Citation for this article: J. Clin. Invest. 116:1823-1825 (2006). doi:10.1172/JCI29172. and 12 months after traumatic injury (3). However, even after these long and arbitrary delays, some patients may exceptionally recover $(4,5)$. In 2002, the Aspen Neurobehavioral Conference Workgroup published the definition and diagnostic criteria of the minimally conscious state (MCS) (6). Patients in MCS will show more than the purely reflex or automatic behav- ior observed in VS survivors, but they will nevertheless be unable to communicate their thoughts and feelings. Recent preliminary evidence indicates that MCS patients demonstrate improvement over a longer period of time and attain better functional recovery as compared with VS patients $(7,8)$. At present, the vast majority of studies on traumatic or ischemic brain damage are focused on the acute phase of coma. This creates a silent epidemic in which there is only minute attention devoted to the long-term diagnostic, prognostic, therapeutic, and social problems of persistent (albeit sometimes transient) disorders of consciousness such as VSs and MCSs $(9,10)$. The number of scientific papers on acute comatose states is about 10-fold higher than the number of publications on chronic disorders of consciousness. Future research efforts should also address the silent epidemic of VS and MCS and so that these challenging neurological states can emerge from the current dark ages of therapeutic nihilism.
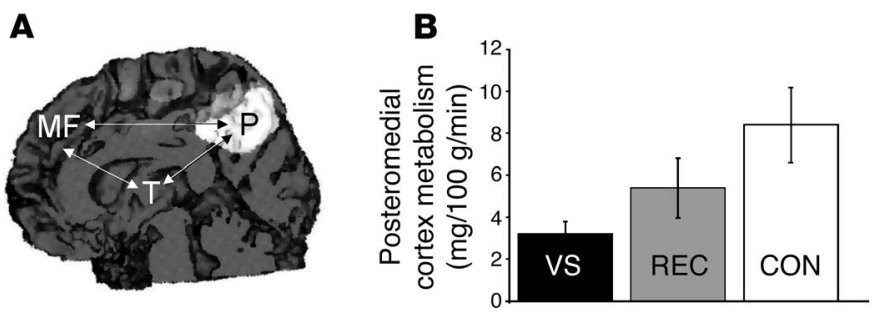

Figure 1

PET studies show that in the rare patients who recover consciousness after being in a chronic VS, glucose metabolism is partially restored in discrete cortical regions. (A) The most significant restoration of glucose metabolism occurs in the posteromedial cortices (shown in white on a medial view of a 3D-rendered MRI). The arrows represent the corticocortical and corticothalamocortical functional disconnections observed in vegetative patients. MF, mesiofrontal cortex; $\mathrm{P}$, precuneus; $\mathrm{T}$, thalamus (nonspecific nuclei). (B) Metabolism in this area is significantly impaired during the VS (black) but resumes near-normal activity after recovery of consciousness (REC; gray). Normal resting metabolic rates of glucose as measured in healthy controls are shown for comparison (CON; white). In this issue of the $\mathrm{JCl}$, Voss and coworkers report on their observation of intracortical connectivity changes in the same area of the brain, as assessed by diffusion tensor MRI, in an exceptional patient who emerged after spending 19 years in an MCS (12). This residual cerebral plasticity in chronic disorders of consciousness has been largely overlooked by the medical community and deserves further study to expose its underlying cellular mechanisms. Figure and data adapted with permission from the Journal of Neurology, Neurosurgery, and Psychiatry (22). 


\section{Understanding "miracle" recoveries from "coma"}

"The brain is a mystery and even more so in these states," says the nurse caring for a beautiful dancer in a VS in Pedro Almodóvar's Academy Award-winning motion picture Habla con ella (Talk to her). Chronic coma (which is much more rare in clinical practice than it is in Hollywood scenarios) and VS or MCS, especially unexpected recovery from these, have always incited the media and public. Several reports in the popular media have described dramatic recovery from "coma." In nearly all reports, recovery of consciousness and function occurred in VS or MCS patients and, in most individuals, recovery occurred within the time frames mentioned above. Incredible cases of recovery reported in the popular media or even the medical literature are often very poorly documented - the nature of the patients' neurologic condition is imprecise, or the timing of the entry into the VS or MCS is extremely atypical (11). In this issue of the JCI, Voss and colleagues present the extraordinary case of recovery from MCS 19 years after traumatic brain injury of a 39-year-old patient (12). The authors have taken the initiative to carefully check this individual's medical history and personally examine this "miracle recovery from coma" case, which was widely covered in the popular media (e.g., see ref. 13). The authors have complemented extensive neuropsychological testing with state-of-the-art structural and functional neuroimaging techniques. Changes in the patient's brain structure and function were compared with measurements obtained in healthy controls and in another MCS patient, the latter not showing recovery after 6 years.

Very few neuroimaging studies have tackled the issue of late recovery of consciousness in severe brain damage. The most remarkable finding in the Voss et al. study (12) was the MRI assessment of transiently increased fractional anisotropy and directionality in the posterior midline cortices (encompassing the cuneus and precuneus), interpreted as increased myelinated fiber densities and novel corticocortical sprouting, paralleling the emergence of the patient from MCS. The same area of the patient's brain also showed amplified metabolic activity, as measured by PET. This finding stresses the importance of the posterior medial structures in consciousness of self and interaction with the environment (14, 15). Activity in the medial parietal cortex (i.e., precuneus) seems to show it to be the brain region that best differentiates MCS from VS patients (16). Interestingly, this area is among the most active brain regions in conscious waking (15) and is among the least active in altered states of consciousness, such as pharmacological coma (17), sleep (18), dementia (19), Wernicke-Korsakoff syndrome, and postanoxic amnesia (20). It has been suggested that this richly connected multimodal posteromedial associative area is part of the neural network subserving human awareness (21).

\section{Searching for the neural correlate of consciousness}

Our center had the opportunity to study neuronal metabolic changes underlying recovery from VS. To date, of 60 patients studied using quantified fluorodeoxyglucose PET, 7 patients recovered consciousness and could be rescanned. In these patients, we localized the brain areas where metabolism was most impaired during the VS and returned to near-normal values after recovery $(22,23)$. Such analyses identified the precuneus (Brodmann areas 7 and $31)$ as the area showing the largest recovery-related metabolic changes (Figure 1), followed by a wide network of frontoparietal associative cortices. In some VS or MCS patients, the disorder of consciousness seems due to a functional "disconnection syndrome" $(16,24)$. Long-range corticocortical (between midline-posterior and latero-frontal areas) and corticothalamic (between midline-posterior cortices and nonspecific thalamic nuclei) disconnections could be identified in a cohort of VS patients, and the rare cases that recovered showed a partial functional restoration of these connections $(22,25)$. The fiber-tracking MRI studies reported by Voss et al. in this issue of the JCI (12) offer indications as to the cellular mechanisms underlying this functional normalization. Their quantification of white matter reorganization shows long-distance rewiring in posterior medial cortices, possibly reflecting axonal sprouting or neurite outgrowth, maybe even related to neurogenesis (known to occur primarily in associative cortices in normal primates) (26).

Severe brain damage represents an immense medical, social, and economic problem that warrants further research. Chronically unconscious or minimally conscious patients present unique problems for diagnosis, prognosis, treatment, and everyday management. They are vulnerable to being denied potentially life-saving therapy if clinical research remains solely focused on the acute stage of the disease. Current functional neuroimaging techniques can prospectively quantify residual neuronal plasticity and the challenging process of recovery. The findings of Voss and coworkers (12) will increase our understanding of severely brain-damaged patients and their "miracle" recovery of consciousness.

Address correspondence to: Steven Laureys, Cyclotron Research Center and Neurology Department, University of Liège, Sart Tilman B30, 4000 Liège, Belgium. Phone: 324-366-23-16; Fax: 32-4-366-29-46; E-mail: steven.laureys@ulg.ac.be.

1. Plum, F., and Posner, J.B. 1966. The diagnosis of stupor and coma. F.A. Davis Co. Philadelphia, Pennsylvania, USA. 197 pP.

2. Jennett, B., and Plum, F. 1972. Persistent vegetative state after brain damage. A syndrome in search of a name. Lancet. 1:734-737.

3. The Multi-Society Task Force on PVS. 1994. Medical aspects of the persistent vegetative state - first of two parts. N. Engl. J. Med. 330:1499-1508.

4. Andrews, K. 1993. Recovery of patients after four months or more in the persistent vegetative state. BMJ. 306:1597-1600.

5. Childs, N.L., and Mercer, W.N. 1996. Late improvement in consciousness after post-traumatic vegetative state. N. Engl. J. Med. 334:24-25.

6. Giacino, J.T., et al. 2002. The minimally conscious state: definition and diagnostic criteria. Neurology. 58:349-353.

7. Whyte, J., et al. 2005. Predictors of outcome in prolonged posttraumatic disorders of consciousness and assessment of medication effects: a multicenter study. Arch. Phys. Med. Rehabil. 86:453-462.

8. Lammi, M.H., Smith, V.H., Tate, R.L., and Taylor, C.M. 2005. The minimally conscious state and recovery potential: a follow-up study 2 to 5 years after traumatic brain injury. Arch. Phys. Med. Rehabil. 86:746-754.

9. Fins, J.J. 2003. Constructing an ethical stereotaxy for severe brain injury: balancing risks, benefits and access. Nat. Rev. Neurosci. 4:323-327.

10. Hirsch, J. 2005. Raising consciousness [editorial]. J. Clin. Invest. 115:1102.

11. The Multi-Society Task Force on PVS. 1994. Medical aspects of the persistent vegetative state - second of two parts. N. Engl. J. Med. 330:1572-1579.

12. Voss, H.U., et al. 2006. Possible axonal regrowth in late recovery from the minimally conscious state. J. Clin. Invest. 116:2005-2011. doi:10.1172/JCI27021.

13. [Anonymous]. Man speaks after 19-year silence. CNN.com/U.S., July 8, 2003. http://edition.cnn. com/2003/US/South/07/07/mute.no.more/.

14. Baars, B., Ramsoy, T., and Laureys, S. 2003. Brain, conscious experience and the observing self. Trends Neurosci. 26:671-675.

15. Gusnard, D.A., and Raichle, M.E. 2001. Searching for a baseline: functional imaging and the resting human brain. Nat. Rev. Neurosci. 2:685-694.

16. Laureys, S., Owen, A.M., and Schiff, N.D. 2004. Brain function in coma, vegetative state, and related disorders. Lancet Neurol. 3:537-546.

17. Alkire, M.T., and Miller, J. 2005. General anesthesia and the neural correlates of consciousness. Prog. Brain Res. 150:229-244.

18. Maquet, P. 2000. Functional neuroimaging of normal human sleep by positron emission tomography. J. Sleep Res. 9:207-231.

19. Salmon, E., Collette, F., Degueldre, C., Lemaire, C., and Franck, G. 2000. Voxel-based analysis of con- 
founding effects of age and dementia severity on cerebral metabolism in Alzheimer's disease. Hum. Brain Mapp. 10:39-48.

20. Aupee, A.M., et al. 2001. Voxel-based mapping of brain hypometabolism in permanent amnesia with PET. Neuroimage. 13:1164-1173.

21. Vogt, B.A., and Laureys, S. 2005. Posterior cingulate, precuneal and retrosplenial cortices: cytology and components of the neural network correlates of consciousness. Prog. Brain Res. 150:205-217.

22. Laureys, S., Lemaire, C., Maquet, P., Phillips, C., and Franck, G. 1999. Cerebral metabolism during vegetative state and after recovery to consciousness [letter]. J. Neurol. Neurosurg. Psychiatry. 67:121-122.

23. Laureys, S., Faymonville, M.E., Moonen, G., Luxen, A., and Maquet, P. 2000. PET scanning and neuronal loss in acute vegetative state. Lancet. 355:1825-1826.
24. Schiff, N.D. 2005. Modeling the minimally conscious state: measurements of brain function and therapeutic possibilities. Prog. Brain Res. 150:473-493.

25. Laureys, S., et al. 2000. Restoration of thalamocortical connectivity after recovery from persistent vegetative state. Lancet. 355:1790-1791.

26. Gould, E., Reeves, A.J., Graziano, M.S., and Gross, C.G. 1999. Neurogenesis in the neocortex of adult primates. Science. 286:548-552.

\title{
Arrhythmogenic right ventricular cardiomyopathy: moving toward mechanism
}

\author{
Calum A. MacRae, ${ }^{1}$ Walter Birchmeier, ${ }^{2}$ and Ludwig Thierfelder ${ }^{2,3}$ \\ ${ }^{1}$ Cardiovascular Research Center, Massachusetts General Hospital and Harvard Medical School, Boston, Massachusetts, USA. \\ 2Max Delbrück Center for Molecular Medicine, Berlin, Germany. ${ }^{3}$ Franz Volhard Clinic, Charité, HELIOS Clinics, Berlin, Germany.
}

\begin{abstract}
Mutations in genes encoding desmosomal proteins have been identified as the major cause of arrhythmogenic right ventricular dysplasia/cardiomyopathy (ARVC), in which the right ventricle is "replaced" by fibrofatty tissue, resulting in lethal arrhythmias. In this issue of the JCI, Garcia-Gras et al. demonstrate that cardiac-specific loss of the desmosomal protein desmoplakin is sufficient to cause nuclear translocation of plakoglobin, upregulation of adipogenic genes in vitro, and a shift from a cardiomyocyte to an adipocyte cell fate in vivo (see the related article beginning on page 2012). This evidence for potential $\mathrm{Wnt} / \beta$-catenin signaling defects sets the scene for a comprehensive exploration of the contributions of this pathway to the pathophysiology of ARVC, not only through perturbation of cardiac patterning and development, but also through effects on myocardial differentiation and physiology.
\end{abstract}

\section{Unique clinical features of ARVC}

Arrhythmogenic right ventricular dysplasia/cardiomyopathy (ARVC) is a heart muscle disorder that predominantly affects the right ventricle and is associated with ventricular tachycardia, syncope, and sudden death (1). With the prevention of lethal arrhythmia, contractile failure is emerging as a major source of morbidity and mortality. At autopsy there is loss of myocardial mass with "replacement" by abnormal adipose and fibrous tissue; in essence a myocardial dystrophy. These features patchily involve the right ventricle and preferentially affect 3 areas: the apex, the inflow tract, and the outflow tract. This distribution may reflect a primary defect in morphogenesis. Although right ventricular disease predominates, the left ventricle is involved in about $50 \%$ of cases,

Nonstandard abbreviations used: ARVC, arrhythmogenic right ventricular dysplasia/cardiomyopathy; PKP2, plakophilin 2.

Conflict of interest: The authors have declared that no conflict of interest exists.

Citation for this article: J. Clin. Invest. 116:1825-1828 (2006). doi:10.1172/JCI29174. and in some individuals there may be a global dilated cardiomyopathy (1).

\section{Human genetics}

ARVC is typically inherited as a dominant Mendelian disease, although recessive variants exist and the involvement of family members often can only be detected by directed screening (1). Human genetic studies over the last few years have offered insight into the potential causes of ARVC. Early work demonstrated substantial genetic heterogeneity, and at least 9 independent loci have now been identified. The discovery of cutaneous and hair follicle involvement in recessive forms of ARVC led to the identification of mutations in the desmosomal proteins plakoglobin and desmoplakin $(2,3)$. These findings also implicated other desmosomal proteins or their partner proteins as candidate causes of the disorder (Table 1). Subsequent work has revealed desmoplakin mutations in a small proportion of dominantly inherited ARVC cases and in arrhythmogenic cardiomyopathy localized to the left ventricle (4-6). The description of mutations in the cardiac ryanodine receptor in families with an exercise-related arrhythmia known as catecholaminergic polymorphic ventricular tachycardia has highlighted phenotypic distinctions from typical ARVC (1).

In the last year, ARVC genetics have taken a significant step forward, led by the observation that mice null for the armadillo protein plakophilin 2 (PKP2), another desmosomal component, die at around E11 with profound cardiac abnormalities (7). These mice fail to form normal cardiac desmosomes, and desmoplakin dissociates from the abnormal junctions accumulating in cytoplasmic aggregates. These findings led in turn to the discovery of dominant mutations in the PKP2 gene in a large proportion of probands with ARVC and not only established mutant desmosomal proteins as a major cause of the syndrome, but also raised the possibility of genetic testing as a diagnostic tool (8). The initial report in a series of 120 unselected European probands identified PKP2 mutations in almost one-third of these individuals (8). Recent data from more selected cohorts of index patients with evidence of familial involvement have suggested that as many as $70 \%$ of such kindreds may have mutations in PKP2 (9). Of note, these investigators also described evidence of founder effects for several PKP2 mutations in remote kindreds, implying less dramatic effects on survival than are seen in other forms of ARVC.

\section{Possible disease mechanisms}

How do these mutant junctional proteins result in a unique, predominantly right ventricular cardiac phenotype? Desmosomal proteins are widely expressed, so the focal nature of apparent pathology in both 